\title{
Cyclic variations in the dynamics of flu incidence in Azerbaijan, 1976-2000
}

\author{
B. D. DIMITROV ${ }^{1}$ AND E. S. BABAYEV ${ }^{2}$ \\ ${ }^{1}$ Academic Unit of Primary Care \& Population Sciences, Faculty of Medicine, University of Southampton, \\ Southampton, UK \\ ${ }^{2}$ Azerbaijan National Academy of Sciences, Baku, Republic of Azerbaijan
}

Received 18 March 2013; Final revision 5 February 2014; Accepted 12 February 2014; first published online 18 March 2014

\section{SUMMARY}

Multicomponent cyclicity in influenza (flu) incidence had been observed in various countries (e.g. periods $T=1,2-3,5-6,8 \cdot 0,10 \cdot 6-11 \cdot 3,13,18-19$ years) and its close similarity with cycles in natural environmental phenomena as meteorological factors and heliogeophysical activity (HGA) suggested. This report aimed at verifying previous results on cyclic patterns of flu incidence by exploring whether flu annual cyclicity (seasonality) and trans-year (13 to $<24$ months) and/or multiannual (long-term, $\geqslant 24$ months) cycles might be present. For this purpose, a relatively long monthly flu incidence dataset consisting of absolute numbers of new cases from the Grand Baku area, Azerbaijan, for the years 1976-2000 (300 months) was analysed. The exploration of underlying chronomes or, time structures, was done by linear and nonlinear parametric regression models, autocorrelation, spectral analysis and periodogram regression analysis. We analysed temporal dynamics and described multicomponent cyclicity, determining its statistical significance. The analysis, considering the flu data specifically stratified in three distinct intervals (1976-1990, 1991-1995, 1996-2000), and also combinations thereof, indicated that the main cyclic pattern was a seasonal one, with a period of $T=12$ months. Further, a number of multiannual cycles with periods $T$ in the ranges of $26-36,62-85$ or $113-162$ months were observed, i.e. average periods of $2 \cdot 5,6 \cdot 1$ and 11.5 years, respectively. Indeed, most of these cycles correspond to similar cyclic parameters of HGA and further analyses are warranted to investigate such relationships. In conclusion, our study revealed the presence of multicomponent cyclic dynamics in influenza incidence by using relatively long time-series of monthly data. The specific cyclic patterns of flu incidence in Azerbaijan allows further, more specific modelling and correlations with environmental factors of similar cyclicity, e.g. HGA, to be explored. These results might contribute more widely to a better understanding of influenza dynamics and its aetiology as well as to the derivation of more precise forecasted estimates for planning and prevention purposes.

Key words: Azerbaijan, influenza incidence, multiannual cyclicity, seasonality, variations.

\footnotetext{
* Address for correspondence: Dr B. D. Dimitrov, MD, MSc, SMHM, DM/PhD, Senior Lecturer in Medical Statistics, Academic Unit of Primary Care \& Population Sciences, Faculty of Medicine, University of Southampton, Level C, South Academic Block, Southampton General Hospital, SO166YD, Southampton, UK.

(Email: b.dimitrov@soton.ac.uk)

Presented in part at the Invited session 'Forecasting \& Time Series Prediction', EURO/INFORMS MMXIII: 26th European Conference on Operational Research, 2013, Rome, Italy.
} 


\section{INTRODUCTION}

Influenza (flu or grippe) is among the most important epidemic diseases. Although preventable to a large extent, it still remains one of the most widely spread infectious diseases globally. Unfortunately, very often the timing, severity and exact strain of influenza remain uncertain. The spread of epidemics and pandemics of infectious diseases depends mainly on individual, physiological or immunological factors as well as on population/social and specific healthcare system phenomena but their appearance might also be related to various meteorological or heliogeophysical factors as part of the natural physical environment [1-4]. The success of preventing influenza will depend largely on well-timed and precise forecasts about its incidence and, possibly, about the subtype of strains appearing by antigenic drift or shift [4]. However, such forecasts in various geographical locations will be incomplete without considering the cyclic components of influenza incidence dynamics, its components and virulence patterns as well as the likely impact of physical environmental factors with similar cyclicity.

Previous studies of annual or monthly incidence and incidence rates across different countries have suggested the existence of high-frequency intrannual (<12 months), seasonal (annual) and low-frequency infrannual, trans-year or multiannual cycles. Transyear cycles are those cycles with periods longer than 12 months, but shorter than 24 months. Various cyclic patterns have been observed, with periods of $1,2-3,5-6$, $8 \cdot 0,10 \cdot 6-11 \cdot 3,13$ and $18-19$ years; some of these have been associated with the influence of cyclic factors from the natural environment [1-6]. Not all of the flu incidence time series have clearly defined linearly increasing or decreasing tendencies but, even so, they could not be easily forecasted ahead by linear trend analysis only. The latter is due to the presence of a high level of variability or volatility. It is possible that variations around such linear trends or mean values may show regular patterns and potentially be exploited in modelling and forecasting with derivation of future estimates and their $95 \%$ confidence intervals (CIs). Being of high public health and socioeconomic importance, the better forecasting of flu incidence variations and their peaks is invaluable for national health systems worldwide. Such forecast estimates may help in identifying populations at increased risk, highlighting the potential for immunization and allow a more adequate allocation of resources. This more precise forecasting will also allow an exploration of risk factors or triggers contributing to the appearance and spread of flu epidemics and pandemics.

Triggers for the increases and/or cyclic variations in flu incidence may be such factors of natural environmental origin as interrelated physico-chemical processes and irradiations of cosmic or terrestrial origin. They are known as 'space weather', i.e. solar, geomagnetic or cosmic ray activity whereas their dynamics show clear cyclic patterns with fluctuations at various frequencies, for instance from hours, days to months, years and tenths of years. Such natural physical activity influences may be also denoted by the common term 'heliogeophysical activity' (HGA). HGA mainly comprises phenomena such as solar activity and geomagnetic field fluctuations but it may also refer to a number of other photic or non-photic events. Such emitting events are sunspots, solar UV radiation, solar wind, $10 \cdot 7-\mathrm{cm}$ solar radio flux, neutron activity, earth-ionosphere cavity/Schumann resonances [7] as indicated or described by quantitative indices such as the sunspot index, geomagnetic indexes (aa, Kp, Ap, Dst) or ozone concentration. Most of these indices exhibit seasonal or circa-annual cyclicity but their chronomes are often multicomponent, with trans-year or multiannual periods such as the 22-year cycle (Hale's solar magnetic cycle) or the 11-year cycle of the sunspot index ( $\mathrm{Rz}$ index or Wolf number). The latter has been a consistent observation over hundreds of years [7-9]. Whether or not such cyclic patterns in flu incidence and HGA are interconnected, remains to be explored.

Previous approaches to flu incidence variations have mostly employed a visualization of cyclic variations $[1,2,4]$, calculation of the number of peaks within pre-defined intervals of time [3, 4] and/or indication of their parallelism to similar variations in cyclic environmental factors [1, 2]. However, other more accurate quantitative approaches in modelling and forecasting flu incidence are needed [5, 8-10]. These methods, with minimal requirements for a specific algorithm and $95 \%$ CIs for the derived estimates and parameters [10-13] should be the following: (1) description, analysis and decomposition of temporal dynamics; nonlinear regression modelling of periodic mode; and derivation and forecasting of cyclic estimates; (2) linear and nonlinear regression modelling to independent variables (e.g. HGA) with such deterministic temporal patterns as seasonality. At present, the relationship of HGA cycles to flu incidence is of increasing importance in the view of the current maximum of the last 11-year solar cycle No. 24, as started in 2008 (http://www.kaltesonne.de/?p=13284). 


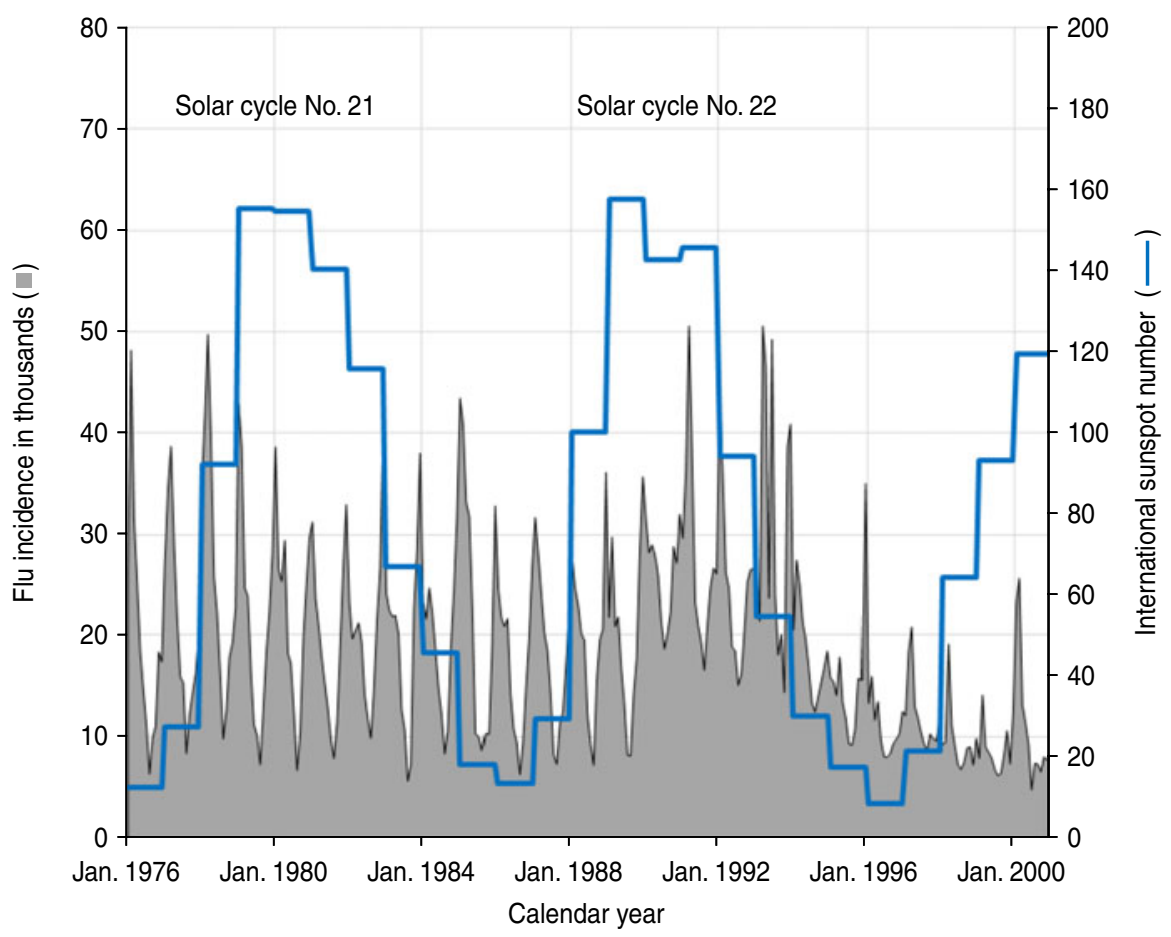

Fig. 1 [colour online]. Dynamics of flu incidence in Grand Baku area, Azerbaijan and annual international sunspot numbers, 1976-2000.

\section{Study objective}

The aims of this study were: (i) to describe the temporal dynamics of monthly flu incidence in Azerbaijan for the years 1976-2000; (ii) to estimate any seasonal and multicomponent infrannual cycles and their eventual relationships with seasonality; and, (iii) to consider flu incidence cyclicity for similarities to and associations with main HGA cycles.

\section{MATERIALS AND METHODS}

\section{Sources and description of time-series datasets}

The time-series datasets consisted of monthly incidence data presented as an absolute number of new cases of influenza (ICD-9-CM, Dx: 487.0-487.8) in the Grand Baku area, Azerbaijan, (population $>3$ million). The incidence data were provided by the Azerbaijani State Institute of Advanced Studies of Doctors, Ministry of Health of the Republic of Azerbaijan. Average monthly and annual values of the international sunspot numbers (ISN) are freely available and were retrieved from the online database of the Solar Influences Data Analysis Center (SIDC) at the Royal Observatory of Belgium (http://sidc.oma.be/).

The flu incidence time series covered the period 1976-2000 (300 months, Fig. 1). The 25-year flu dataset, spanning over more than two 11-year cycles of solar activity (No. 21 and No. 22), was divided into three main intervals: interval 1 (1976-1990, $n=180$ months), interval 2 (1991-1995, $n=60$ months) and interval 3 (1996-2000, $n=60$ months). This stratification was necessary to reflect the underlying demographic and socio-political changes that occurred during this period. The first interval was characterized by relative stability, with full medical care coverage (the so called 'Soviet' period, with obligatory healthcare); the second interval - by economic downturn due to the collapse of the USSR as characterized by difficulties and malfunctioning of the healthcare system; and the third interval-by further recovery and stabilization of the modern national healthcare system. All datasets are from public sources and can be obtained from the authors upon request.

\section{Statistical analyses}

Different statistical methods for time-series analyses and modelling were used (Table 1). Descriptive statistics with linear and nonlinear regression modelling over time were applied. As a second step, autocorrelation and spectral functions for amplitude and density were explored to examine whether the variations may exhibit cyclic patterns (Figs 2, 3). Last, a periodogram 
Table 1. Complex statistical approach to reveal and model cyclic patterns in incidence time series

\begin{tabular}{|c|c|c|}
\hline Statistical method & Purpose & Confidence test \\
\hline $\begin{array}{l}\text { 1. Descriptive statistics } \\
\text { (trend analysis) }\end{array}$ & $\begin{array}{l}\text { Determines a tendency within time series. It aims } \\
\text { at tracking more closely the original dynamics } \\
\text { (either cyclic or not) by univariate linear } \\
\text { or nonlinear regression models }\end{array}$ & $\begin{array}{l}\text { One-sample confidence tests at } P=0.05 \\
(F \text { test, Student's } t \text { statistic, } z \text { statistic })\end{array}$ \\
\hline $\begin{array}{l}\text { 2. Autocorrelation/ } \\
\text { spectral analyses }\end{array}$ & $\begin{array}{l}\text { Describes patterns of variations within time } \\
\text { series. It helps to reveal whether variations } \\
\text { in time series might be cyclic or not } \\
\text { (moving average, e.g. ARMA/ARIMA models } \\
\text { or Bartlett 'windows' for spectral amplitude/ } \\
\text { density estimates can also be used, see } \\
\text { Figs } 2 \text { and 3) }\end{array}$ & $\begin{array}{l}\text { Confidence tests for correlation coefficient at } \\
P<0.05 \text { (Student's } t \text { statistic, } z \text { statistic) }\end{array}$ \\
\hline $\begin{array}{l}\text { 3. Periodogram } \\
\text { regression analysis } \\
\text { (PRA) }\end{array}$ & $\begin{array}{l}\text { More precisely defines the type of variations } \\
\text { within time series. It determines the length of } \\
\text { the cycle with its amplitude and their statistical } \\
\text { significance (e.g. Fig. 4) }\end{array}$ & $\begin{array}{l}\text { Confidence tests for correlation coefficient at } \\
P<0.05 \text { (Student's } t \text { statistic, } z \text { statistic, } F \text { test); } \\
\text { additional testing against Gaussian random } \\
\text { 'red' noise can also be applied }[5,8,9]\end{array}$ \\
\hline
\end{tabular}

(a) Autocorrelation of flu incidence

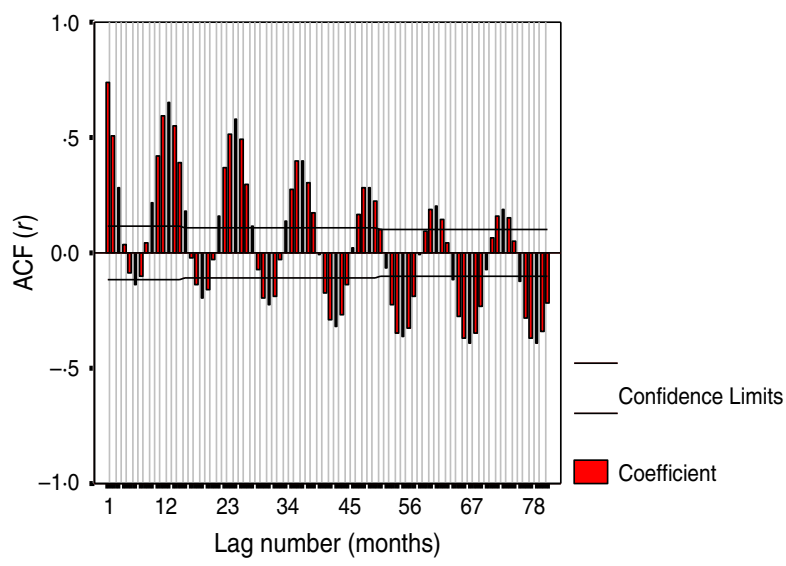

(b) Partial autocorrelation of flu incidence

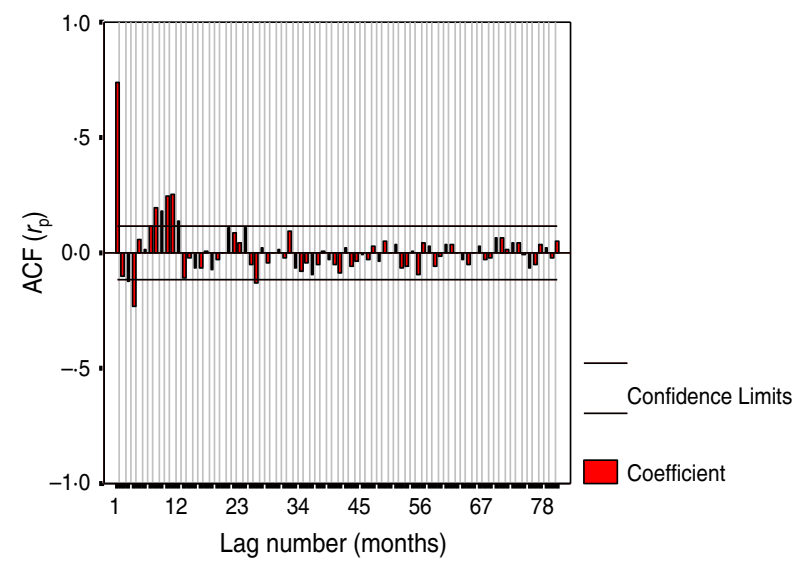

Fig. 2 [colour online]. Autocorrelation analysis of flu incidence in Azerbaijan, 1976-2000. (a) Clear autocorrelation $(r)$ peaks are seen where the lag number is in multiples of $\approx 12$ months. $(b)$ Partial autocorrelation function $\left(r_{\mathrm{p}}\right)$ indicates that the strongest influence on monthly flu incidence was from the previous month (lag number $=1$ month, $r=0 \cdot 74)$ and $\sim 1$ year before (lag number peak at 11 months, $r_{\mathrm{p}}=0 \cdot 25$ ); a tendency of autocorrelation at 26 months may also exist $(r=-0 \cdot 13)$.

regression analysis (PRA) with determination of the cycle length and its statistical significance at $P<0.05$ was applied [5, 11-14]. PRA, using the sigma method for testing the statistical significance of the linear correlation coefficients, was successfully applied in earlier studies on helioepidemiology of various cancers and infectious diseases [5,11-13]. In this work we approximated the studied time series $f(t)$ with a number of minimizing correlation-regression functions $F(t)$ of periodic mode:

$F(t)=a_{0}+A \cos \frac{2 \pi t}{T}+B \sin \frac{2 \pi t}{T}$, where $a_{0}$ is the mean of $f(t)$ and $T$ is a preliminary fixed, stepwise increasing period. The parameters $A$ and $B$ are coefficients of regression obtained by least-square calculations for the actual $f(t)$ at each $T$; $t$ is the current moment of time (a serial number of the month: $0,1,2,3, \ldots, N-1$ ), and $N$ is the total number of values in the time-series sample $[9,11,15]$. A range of the linear coefficients $R$ is constructed against each of the tested periods $T$ in months (Fig. 4) as a 'periodogram' of the time series, i.e. power in the period domain. The PRA with the use of basic, i.e. original, or detrended or decycled time series allows a description of the so-called 
(a) Original spectrum of variations in flu incidence

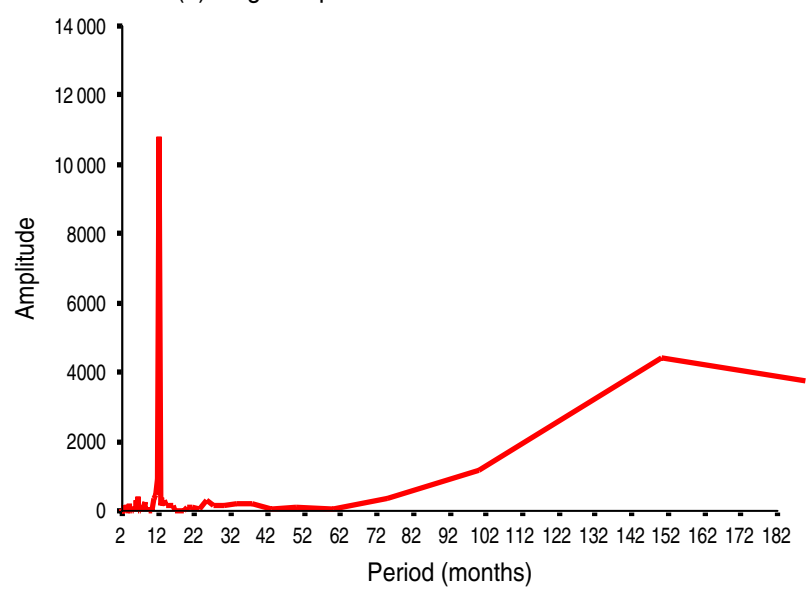

(b) Smoothed spectrum of variations in flu incidence

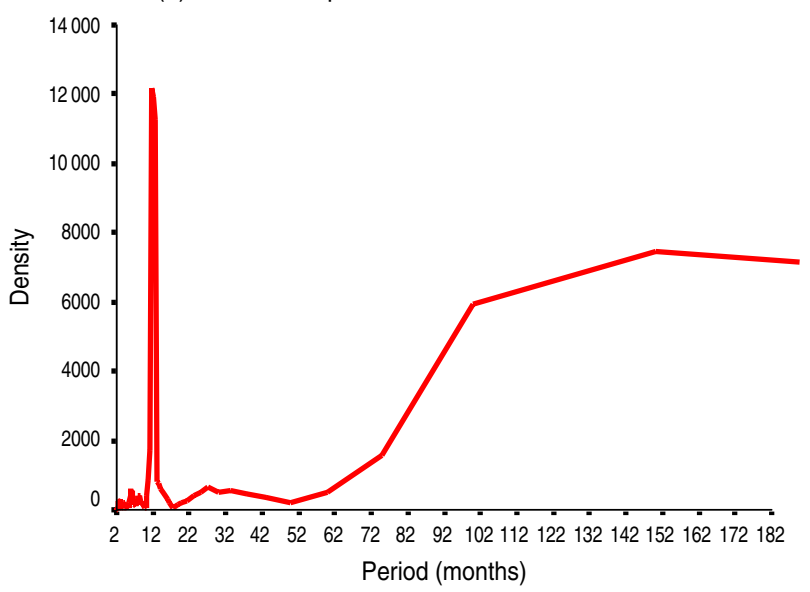

Fig. 3 [colour online]. Spectral analysis of flu incidence in Azerbaijan, 1976-2000. (a) The unsmoothed spectrum of amplitudes against the period indicated two main peaks at 12 and 152 months (spectral windows on a log scale of 10-18, 22-28 and 100-200 months, dara not shown). (b) The smoothed spectrum, where irregular variations have been removed, has confirmed the main peaks at similar periods of 12 and 150 months and a small, additional peak at 27 months (spectral windows on a $\log$ scale of 10-16, 22-30 and 50-200 months, data not shown). Of note, periods $>150$ months may represent artefacts or long-term trends in time series of 300 months where detrending/decycling was not applied a priori, therefore, such peaks should be viewed only as informative in nature and be interpreted with caution.

'hypercycles' on the periodogram. A 'hypercycle' is denoted when the length of its period $T_{\mathrm{H}}$ exceeds the length of the analysed time-series set [16]. It is possible that such hypercycles represent or are closely related to the long-term linear or nonlinear trends, therefore the time series can be further decomposed. Then, the period/s $T_{\mathrm{H}}$ can be removed later by decycling, a type of 'detrending' of the time series. The hypercycles may be also included in reconstructing and forecasting the incidence estimates by a trigonometric approximation when they are indicated to improve the forecasting power of the cyclic models [13, 15]. SPSS 15 (SPSS Inc., USA), IBM SPSS Statistics 21 (IBM Corporation, USA) and a specialized package for time-series analyses '6D-STAT' were used. The software '6D-STAT' (www.astro.bas. bg/ komitov/dataproc.htm) was kindly provided by B. Komitov (Stara Zagora, Bulgaria).

\section{RESULTS}

\section{Descriptive (exploratory) analyses}

Descriptive analyses have shown that flu incidence in the Grand Baku area has remained mainly stable or slightly decreased during the study period from 1976 to 2000. However, the linear or nonlinear regression models by various functions [13] have only been able to explain about $3-61 \%$ of the temporal variations. The best models were nonlinear, mainly of cubic,
S-shaped or power/exponential type (data not shown). Notably, a minimum $39 \%$ of the variations remained unexplained even by decreasing tendencies. Such variations have indicated that analysing flu incidence by such trend models alone, even of nonlinear shape, will be of little use. We then postulated that the temporal distribution of flu incidence may be better represented by other nonlinear functions with high-frequency (seasonal, intrannual) and/or longer, low-frequency (trans-year, multiannual) cyclic components $[10,13,15]$.

\section{Autocorrelation analysis}

To provide evidence on the existence of cyclic variations, we constructed and analysed the autocorrelograms of flu incidence (Fig. 2). The autocorrelation function (ACF, Fig. 2a) and partial autocorrelation function (Fig. 2b) suggested that multiple cyclic components might co-exist with peaks in the windows of 1-22 and 64-92 as well as 132-156 months (data not shown). The main components of these windows are lag numbers, or cycles of 1 month showing dependence on the previous month, as well as for the previous 4 and 11 months ( $\approx$ seasonal). Infrannual cycles of 82,85 and 89 months were also detected (data not shown). Due to inherent limitations of the method where autocorrelations may only be evident when the low-frequency cycles are multiples of prevailing 12-month or other high-frequency 


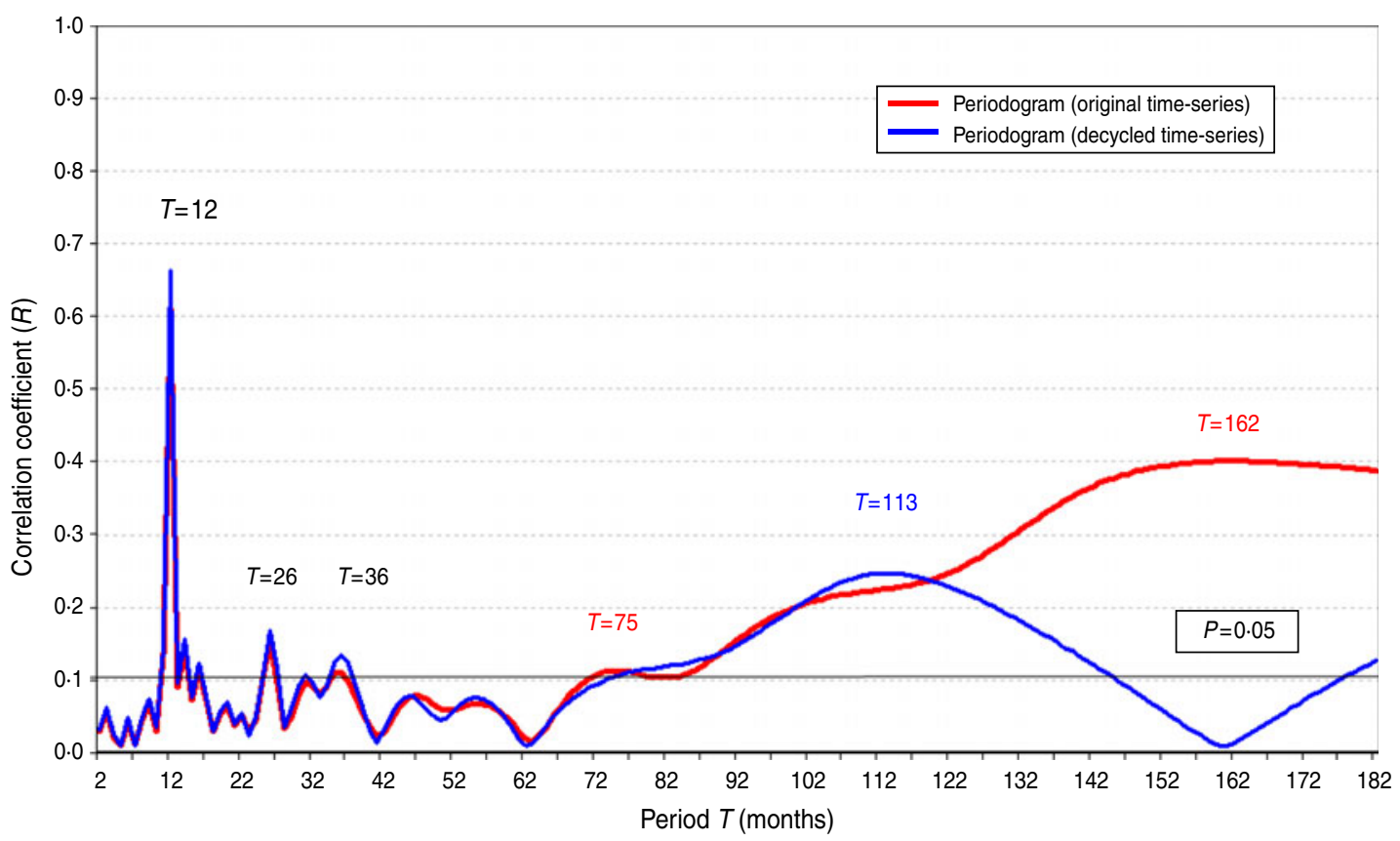

Fig. 4 [colour online]. Periodogram regression analysis (PRA) of cyclic patterns in the variations of flu incidence in Azerbaijan, 1976-2000. The periodograms show correlation coefficients $R$ against period $T$ (months). The peaks above the horizontal line indicate the significant periods at $P<0.05\left(P=0.05\right.$ for a theoretical $R_{\mathrm{T}}=0.11$ at variance $\left.F \approx 2 \sigma, n=300\right)$ for original (red curve) and decycled/detrended (blue curve) time series. $Y$ axis: correlation coefficient of $R$ for flu incidence variations (the periods $T=12,26$ and 36 months are common for both time series in black font; periods $T=75$ and 162 months are observed in the original in red font, while the period $T=113$ months is secondary and it appears only in the decycled time-series, in blue font). (See Table 2 for more details.)

components, to further confirm these patterns, a spectral analysis of flu incidence dynamics was also applied (Fig. 3).

\section{Spectral analysis}

To test above conjectures and better define the main existing cycles, we obtained spectral amplitudes and densities (Fig. 3). We described again the main 12-month cycle and provided further evidence on infrannual cycles of 27 months $(2 \cdot 3$ years, Fig. $3 b)$, 102 months $(8 \cdot 5$ years, Fig. $3 a)$ and, possibly, $\approx 150$ months $(12.5$ years, Fig. $3 a, b)$. The latter may represent an artefact or a long-term trend in the flu incidence time series where detrending/decycling was not applied a priori. To further quantify and statistically confirm the above cyclic patterns, a periodogram analysis with correlation-regression function of periodic mode (see the Materials and Methods section for more details) was also applied (Fig. 4, Table 2).

\section{Periodogram regression analysis}

To better quantify and apply formal statistical confidence testing to the underlying cyclic patterns in flu incidence dynamics, we constructed periodograms (Fig. 4) and confirmed the main cycle of 12 months. This period appears to be the most important and repeated cyclic pattern across all stratifications. Beyond seasonality, several multiannual cycles with periods $T$ in the ranges of 26-36, 62-85 and 113-162 months were also established, with an average period for $T$ of $2 \cdot 5,6 \cdot 1$ and 11.5 years, respectively. For some of the intervals, one or more hypercycles were described. These may represent or be related to longterm nonlinear trends with periods $T_{\mathrm{H}}=128,156$, 194 and 208 months (i.e. hypercycles of 10·7, 13.0, $16 \cdot 2$ and $17 \cdot 3$ years, respectively; data not shown). PRA is now illustrated by depicting only the periodograms for the whole study period (1976-2000). All results for the different intervals and their combinations are summarized in Table 2.

Where an additional elaboration of the time series was considered appropriate in order to control for revealed cyclic patterns in the original time series, a decomposition and further decycling were applied. When removing the cycle of 162 months or a de-trending were applied, e.g. a new time series was derived and an additional periodogram was constructed again 
Table 2. Cyclic patterns of variations in flu incidence time series in Grand Baku area, Azerbaijan (1976-2000)

\begin{tabular}{|c|c|c|c|c|c|c|}
\hline $\begin{array}{l}\text { Calendar time } \\
\text { (intervals) }\end{array}$ & Pattern & $\begin{array}{l}\text { Period } T \\
\text { (months) }\end{array}$ & $R$ & $p_{\mathrm{R}}{ }^{*}$ & $n$ (months) & Time span \\
\hline 1. Interval 1 & $\begin{array}{l}\text { Cycle } \\
\text { Cycle } \\
\text { Cycle } \\
\text { Hypercycle } \dagger\end{array}$ & $\begin{array}{r}12 \cdot 0 \\
29 \cdot 0 \\
75 \cdot 0 \\
194 \cdot 0\end{array}$ & $\begin{array}{l}0 \cdot 80 \\
0 \cdot 15 \\
0 \cdot 17 \\
0 \cdot 14\end{array}$ & $\begin{array}{l}<0.003 \\
<0.037 \\
<0.009 \\
<0.054\end{array}$ & 180 & 1976-1990 \\
\hline 2. Interval 2 & $\begin{array}{l}\text { Cycle } \\
\text { Cycle } \\
\text { Cycle } \\
\text { Hypercycle }\end{array}$ & $\begin{array}{r}8 \cdot 0 \\
12 \cdot 0 \\
33 \cdot 5 \\
128 \cdot 0\end{array}$ & $\begin{array}{l}0 \cdot 29 \\
0 \cdot 51 \\
0 \cdot 44 \\
0 \cdot 46\end{array}$ & $\begin{array}{l}<0.013 \\
<0.003 \\
<0.003 \\
<0.003\end{array}$ & 60 & 1991-1995 \\
\hline 3. Interval 3 & $\begin{array}{l}\text { Cycle } \\
\text { Cycle } \\
\text { Cycle } \\
\text { Cycle }\end{array}$ & $\begin{array}{r}6 \cdot 0 \\
12 \cdot 0 \\
17 \cdot 0 \\
27 \cdot 0\end{array}$ & $\begin{array}{l}0 \cdot 29 \\
0 \cdot 64 \\
0 \cdot 44 \\
0 \cdot 28\end{array}$ & $\begin{array}{l}<0.016 \\
<0.003 \\
<0.003 \\
<0.021\end{array}$ & 60 & 1996-2000 \\
\hline $\begin{array}{l}\text { 4. Intervals } 1+2 \\
\text { (combined) }\end{array}$ & $\begin{array}{l}\text { Cycle } \\
\text { Cycle } \\
\text { Cycle } \\
\text { Cycle } \\
\text { Cycle }\end{array}$ & $\begin{array}{r}12 \cdot 0 \\
26 \cdot 5 \\
36 \cdot 0 \\
85 \cdot 0 \\
158 \cdot 0\end{array}$ & $\begin{array}{l}0 \cdot 71 \\
0 \cdot 16 \\
0 \cdot 18 \\
0 \cdot 24 \\
0 \cdot 21\end{array}$ & $\begin{array}{l}<0.003 \\
<0.011 \\
<0.004 \\
<0.003 \\
<0.003\end{array}$ & 240 & 1976-1995 \\
\hline $\begin{array}{l}\text { 5. Intervals } 2+3 \\
\text { (combined) }\end{array}$ & $\begin{array}{l}\text { Cycle } \\
\text { Cycle } \\
\text { Cycle } \\
\text { Hypercycle } \\
\text { Hypercycle }\end{array}$ & $\begin{array}{r}12 \cdot 0 \\
26 \cdot 0 \\
62 \cdot 0 \\
156 \cdot 0 \\
208 \cdot 0\end{array}$ & $\begin{array}{l}0 \cdot 38 \\
0 \cdot 23 \\
0 \cdot 22 \\
0 \cdot 69 \\
0 \cdot 70\end{array}$ & $\begin{array}{l}<0.003 \\
<0.009 \\
<0.012 \\
<0.003 \\
<0.003\end{array}$ & 120 & 1991-2000 \\
\hline $\begin{array}{l}\text { 6. Intervals } 1+2+3 \\
\text { (combined) }\end{array}$ & $\begin{array}{l}\text { Cycle } \\
\text { Cycle } \\
\text { Cycle } \\
\text { Cycle } \\
\text { Cycle }\end{array}$ & $\begin{array}{r}12 \cdot 0 \\
26 \cdot 0 \\
36 \cdot 0 \\
75 \cdot 0 \\
162 \cdot 0\end{array}$ & $\begin{array}{l}0 \cdot 61 \\
0 \cdot 15 \\
0 \cdot 14 \\
0 \cdot 11 \\
0 \cdot 40\end{array}$ & $\begin{array}{l}<0.003 \\
<0.008 \\
<0.050 \\
<0.050 \\
<0.003\end{array}$ & 300 & 1976-2000 \\
\hline
\end{tabular}

* Statistical significance of the correlation coefficient $R$ is denoted by $p_{\mathrm{R}}$ after the $z$ test at $P<0 \cdot 05$ when $R>1 \cdot 96 S_{\mathrm{R}}\left(z=R / S_{\mathrm{R}}\right)$, where $S_{\mathrm{R}}$ is the standard error of $R$ [9]; $R$, correlation coefficient from the periodogram (i.e. PRA, periodogram regression analysis) indicating a peak at a particular period $T$ of an underlying cyclic pattern (e.g. see periodograms in Fig. 4); $p_{\mathrm{R}}$, statistical significance of $R ; n$, sample size of time series (months).

$\dagger$ Hypercycle (long-term cyclic pattern) is revealed when the period $T_{\mathrm{H}}$ is longer than the sample size of the time series (see Dimitrov et al. [16] for further references on hypercyclicity).

(e.g. see the blue curve in Fig. 4). It can be observed that after this procedure the three cycles with periods $T=12,26$ and 36 months remained significant. At the same time, while the cycles of 75 and 162 months disappeared, a new (secondary) cycle of 113 months appeared in the derived time series. Therefore, it can be suggested that the high-frequency cyclicity (annual and, possibly, short-term multiannual periods of 2-3 years) was not dependent upon the long-term nonlinear decreasing trend. Seasonality appears to be an independent, stand-alone stable cyclic pattern over the whole study period of 300 months. Of note, the main cyclic components of monthly flu incidence in Azerbaijan (1, 2-3, 6-7, 10-13, 16-17 years) may be considered similar to some of the most prominent cyclic components in such global HGA parameters as the sunspot index, F10.7 solar radio flux, solar UVR, aa index, $\mathrm{K}$ index and Schumann resonance modes, among others.

\section{DISCUSSION}

Descriptive, autocorrelation and regression analyses have shown clearly that cyclic patterns (beyond seasonality) may exist in the variations of time series of flu incidence in Azerbaijan over the years 1976-2000 ( $n=300$ months). First, we found that despite the extensive analysis and modelling, the usual linear or nonlinear models accounted only for up to $61 \%$ of overall variance. The remaining, minimum $39 \%$ variability was unaccounted for; moreover, the latter appeared most likely in cycles. Notably, the 
predominant cycles in flu incidence variations, beyond the seasonal one, are those having periods of $2-3,6-7,10-13$ and $16-17$ years. This is very interesting since a relatively recent study [5] has shown that main cycles of 2.25-2.50 and 18-19 years exist in flu annual incidence rates (Bulgaria, 1941-1986, $n=46$ years), thereby confirming earlier reports by Chizhevsky [1] and Sidyakin et al. [2] on the existence of short-term multiannual cycles of 2-3 years in flu dynamics.

Some of the earlier studies on influenza incidence dynamics also postulated interesting patterns of cyclic appearance of flu incidence, with peaks at specific points along the 11-year solar activity cycle in the 20th century [1-4]. Reports of flu pandemics, occurring around the sunspot maxima, were later scrutinized by Ertel [6]. Ertel had reanalysed data on 25/42 'claimed' pandemics during 1700-1985 (total $n=286$ years) and suggested that their peaks tended to occur in years near the minima of these solar cycles but only during the 18 th century. This relationship had probably declined over time thus becoming more complex during the 19th and 20th centuries, possibly showing unstable historical patterns. Another explanation might be, as suggested earlier by Chizhevsky [1], that flu incidence peaks (at least for 17th-19th centuries or earlier) appeared most likely on the ascending or descending slopes of the 11-year solar cycle. This means that the incidence peaks are most likely preceding and/or following the solar maxima by $1-2$ years, probably influenced through the cyclic geomagnetic disturbances having double peaks, just before and after the peak of sunspot activity.

Sidyakin and colleagues [2] also reported that flu death epidemic changes had been $1 \cdot 3$ times more frequent in the years of sudden increase in solar activity. They found that $42 / 44 \mathrm{flu}$ death epidemics coincided with such intervals of sudden change. These authors also suggested that the cyclic variations of 2-4 years in flu epidemics could be associated with the frequency of such sudden changes, i.e. the so called 'reference points' along the 11-year sunspot cycle. Recent calculations indicated that such temporal appearances of flu epidemics [17] were very likely when taking into account all cycles together since the year $1700-$ Tapping and collaborators [17] clearly illustrated by phase offset that five epidemic peaks occurred from 'minimum' to 'minimum' (inclusive) along the 11-year sunspot cycle.

Notably, we can assume that the main short-term multiannual (biennial/triennial) cycle in flu variation is the cycle with a period $T=2-3$ years [5], that is, beyond the prevailing seasonality and irrespective of the geographical location, i.e. similarly to malignant melanoma of the skin [18]. Therefore, any other hierarchy of such cyclicity with a lower frequency of 6-7 years or 10-13 years may be considered [2] as a 'repetition' of this principal, common period appearing along the curve of the 11-year sunspot cycle. Indeed, it is possible that for a given region flu incidence oscillates in several waves contemporaneously but in different phases. It is theoretically plausible that after a defined number of cycles or calendar years, the seasonally determined peak within the particular year may reach the highest possible level when all other close, shorter infrannual minor epidemic waves of 2-3 years or 5-6 years coincide. Such coincidences, or phase-locked temporal occurrences may certainly give rise to higher flu rates. These higher flu rates may express themselves with a major peak of a longer, infrannual flu cycle with a period $T=10-11$ years [19] or $T=18-19$ years [5]. Unfortunately, we are not able to further elucidate if such rises of major flu epidemics are due to one single flu strain causing the above-mentioned minor epidemics or if this is an appearance of new strains/variants (e.g. shift, drift), or a combination of both possible scenarios.

We cannot strongly conclude on the likelihood of HGA-related aetiological mechanisms of flu epidemic/ pandemic peaks. However, if we consider about four minor epidemics during one average solar cycle, i.e. at the minimum, ascending slope, maximum and descending slope [17], a role may be postulated for the solar UV radiation that clearly fluctuates along the 11-year cycle with high frequency $[1,2]$. More recent publications have suggested a role for both UV radiation and vitamin $\mathrm{D}$ formation in the prevention of colds and flu [20]. Therefore, minimum solar UV radiation, and possibly, a subsequent vitamin D decrease during the solar minima as well as at the solar maxima $[1,2]$ may easily facilitate the development and spread of influenza concomitantly, at about the same time intervals, but in different geographical locations. Being novel historically, at least in Britain as hypothesized still in 1940 [21], and also more recently suggested for Azerbaijan [22], these may be considered as very important conjectures. The temporal analyses and correlations between disease incidence and solar activity factors have been shown to be very useful in generating hypotheses and predictive models not only in influenza but also in other infectious diseases 
such as cholera [23]. It is of interet to quote here Dr Douglas Webster [21], who wrote:

this and the often close correspondence between the monthly sun-spot oscillations and influenzal 'waves' (as, for instance, in the three pandemic waves of 1918-19) suggest the possible causal relationship of minor sun-spot cycles to cycles of virus activity in influenza.

To what extent such infrannual cyclicity of $2-3$ years in flu incidence from the Grand Baku area in Azerbaijan is associated, or may interact with the underlying seasonality, as a main annual pattern is still largely unknown and may represent an interesting research question for further studies.

In conclusion, we summarize that by using a unique and relatively long time series of monthly data, we revealed a complex, multicomponent dynamics of flu incidence in Grand Baku area, Azerbaijan for the years 1976-2000. First, the main cyclic pattern was a seasonal period $T=12$ months. Second, against the background of a long-term nonlinear decreasing trend, multiannual cyclicity with periods $T=26-36$, 62-85 or 113-162 months was also described (average periods $\approx 2 \cdot 5,6 \cdot 1$ and 11.5 years, respectively). The cyclicity of 2-3 years was found to be the most interesting. Third, we established that most of these cycles correspond to similar HGA cycles and further analyses are warranted to investigate such relationships. Last, but not least, the specific cyclic patterns of flu incidence in Azerbaijan may not only advance our understanding of influenza aetiology, but may also contribute to the derivation of more precise forecasts for prevention and public healthcare purposes.

\section{ACKNOWLEDGEMENTS}

The authors thank Professor Dr F. E. Sadykhova, Azerbaijani State Institute of Advanced Studies of Doctors named after A. Aliyev, Ministry of Health, Republic of Azerbaijan, for providing the data on influenza and her helpful comments during the preparation of this manuscript. Special thanks are due also to Professor G. Cornelissen and the specialists from The Halberg Chronobiology Centre, University of Minnesota, USA, for their help and assistance provided during the revision of the manuscript. This study was initiated in 2009 when Dr Dimitrov was with the HRB Centre for Primary Care Research at the Royal College of Surgeons in Ireland.

\section{DECLARATION OF INTEREST}

None.

\section{REFERENCES}

1. Chizhevsky AL. Terrestrial Echo of Solar Storms [in Bulgarian]. Sofia: Nauka i Izkustvo, 1984, pp. 123-142.

2. Sidyakin VT, et al. Cosmic Ecology [in Russian]. Kiev: Naukova Dumka Press, 1985, pp. 67-69.

3. Hope-Simpson RE. Sunspots and flu: a correlation. Nature 1978; 275: 86.

4. Hoyle F, Wickramasinghe NC. Sunspots and influenza. Nature 1990; 343: 304.

5. Dimitrov BD, Komitov BP, Dimitrova BS. Analysis of incidence variations of some diseases in Bulgaria during XX century. Possible effect of solar activity [in Bulgarian with English Abstract]. Higiena $i$ Zdraveopazvane 1990; 33: 9-14.

6. Ertel S. Influenza pandemics and sunspots - easing the controversy. Naturwissenschaften 1994; 81: 308-311.

7. Cherry N. Schumann resonance and sunspot relations to human health effects in Thailand. Natural Hazards 2003; 29: 1-11.

8. Valev DM. Relationship between the atmospheric pressure in the time of the warm half-year in Sofia and Hale's solar cycle [in Russian]. Solnechnie Dannye 1986; 3: 75-79.

9. Komitov B. The 'Sun-climate' relationship. I. The sunspots and the climate. Bulgarian Astronomical Journal 2009; 11: 139-151.

10. Dimitrov BD, Atanassova PA, Rachkova MI. Cyclicity of suicides may be modulated by internal or external ₹11-year cycles: an example of suicides rates in Finland. Sun \& Geosphere 2009; 4: 50-54.

11. Dimitrov BD. Heliophysical activity and incidence variations of skin malignant melanoma melanoma in Czechoslovakia: a regional study. International Journal of Biometeorology 1993; 37: 68-71.

12. Dimitrov BD, et al. Cyclic patterns of cerebral malaria admissions in Papua New Guinea for the years 1987-1996. Epidemiology and Infection 2013; 141: 2317-2327.

13. Dimitrov BD. Cyclic patterns of incidence variations for stomach cancer in the North-Western Region of England. Croatian Medical Journal 2000; 41: 197-202.

14. Dimitrov BD. Cyclicity in incidence variations of meningococcal infections in Bulgaria is similar to that of solar activity. Central European Journal of Public Health 2000; 8: 114-116.

15. Dimitrov BD. Cyclicity of variations in the frequency of suicides in Bulgaria. Medicina Legalis Baltica 1999; 8: $26-31$.

16. Dimitrov BD, Shangova-Grigoriadi S, Grigoriadis E. Cyclicity in variations of incidence rates for breast cancer in different countries. Folia Medica (Plovdiv) 1998; 40 (Suppl. 3B): 66-71.

17. Tapping KF, Mathias RG, Surkan DL. Influenza pandemics and solar activity. Canadian Journal of Infectious Diseases 2001; 12: 61-62.

18. Dimitrov BD, Rachkova MI, Atanassova PA. Cyclic patterns of incidence rate for skin malignant melanoma: 
association with heliogeophysical activity. Journal Zhejiang University Science B 2008; 9: 489-495.

19. Kilbourne ED. An explanation of the interpandemic antigenic mutability of the influenza virus. Journal of Infectious Diseases 1976; 128: 668.

20. Cannell JJ, et al. Epidemic influenza and vitamin D. Epidemiology and Infection 2006; 134: 1129-1140.

21. Douglas Webster JH. The periodicity of sun-spots, influenza and cancer. British Medical Journal 1940; 2: 339.
22. Babayev ES, et al. An influence of the heliogeophysical conditions on influenza diseases in Azerbaijan during 1976-2000. In: Maris G, Messerotti M, eds. Observations Solaires. Bucharest: Editions de l'Academie Roumaine, 2002, pp. 37-40.

23. Gumarova L, et al. Geographically selective assortment of cycles in pandemics: meta-analysis of data collected by Chizhevsky. Epidemiology and Infection 2012; 141: 2173-2184. 